06

\title{
Моделирование генерации гамма-излучения при взаимодействии сильноточных пучков ультрарелятивистских частиц с плазмой
}

\author{
(C) А.С. Самсонов, И.Ю. Костюков
}

Институт прикладной фризики РАН, 603155 Нижний Новгород, Россия

e-mail: asams@ipfran.ru

Поступила в редакцию 20.12.2021 г.

В окончательной редакции 20.12.2021 г.

Принята к публикации 30.12.2021 г.

Рассмотрено образование жёстких фотонов в процессе взаимодействии сильноточных пучков ультрарелятивистских частиц с протяжёнными плазменными мишенями. С помощью полноразмерного трёхмерного численного моделирования методом частиц-в-ячейках установлена зависимость эффективности конверсии энергии пучка в энергию гамма-излучения от параметров мишени: толщины и концентрации. Представлена аналитическая оценка, полученная в рамках приближённой модели, хорошо совпадающая с результатами численного моделирования. Рассмотренная конфигурация взаимодействия может являться достаточно простым и эффективным способом получения качественных гамма-пучков.

Ключевые слова: сильноточные пучки, плазменные мишени, гамма-излучение.

DOI: $10.21883 /$ OS.2022.04.52268.56-21

В настоящее время изучение физики сильных полей в основном связано с использованием мультипетаваттных лазерных установок, таких как ELI [1], SULF [2], Apollon [3], а в будущем 100-ПВт-лазеров (XCELS [4], SEL [5] и т.д.). Однако достижение всё больших значений интенсивности предъявляет все более жесткие требования к контрастности, стабильности, качеству импульсов и т.д. [6]. В связи с этим сильноточные ускорители частиц, отличающиеся высоким качеством пучка и стабильностью, могут стать привлекательной альтернативой для экспериментов в области физики сильных полей. В последнее время плазменные методы ускорения рассматриваются как перспективное направление для создания компактных линейных ускорителей [7]. Создание такого ускорителя является главной целью проекта FACET-II [8-10]. Пучки заряженных частиц в таких ускорителях могут генерировать сильные электромагнитные поля, что делает возможным при столкновении таких пучков с веществом (или другими пучками) наблюдение таких процессов физики сильных полей, как генерация гамма-излучения в процессе нелинейного комптоновского рассеяния [11-13], рождение электрон-позитронных пар $[14,15]$ или даже эффекты непертурбативной квантовой электродинамики (КЭД) $[9,16,17]$.

Ожидается, что на установке FACET-II будут получены пучки с плотностью электронов свыше $10^{29} \mathrm{~m}^{-3}$, что соответствует характерной концентрации электронов в твердом веществе. При распространении такого пучка в твердом теле может возбуждаться сильно нелинейная кильватерная волна - „баббл“, образование которого обычно рассматривается в гораздо менее плотных средах, например в газе $[18,19]$.
В данной работе с помощью полноразмерного трёхмерного численного моделирования методом частицв-ячейках (PIC) изучается процесс генерации гаммафотонов при взаимодействии пучка ультрарелятивистских электронов с толстой плазменной мишенью. Рассматривается зависимость эффективности конверсии энергии пучка в энергию гамма-излучения от параметров мишени. Численное моделирование было выполнено с помощью PIC-кода QUILL [20], в котором образование вторичных частиц учитывается с помощью метода Монте-Карло. Для численного решения уравнений Максвелла была использована гибридная схема, описанная в публикации [21], которая позволяет существенно уменьшить инкремент численной черенковской неустойчивости [22-24]. Параметры пучка были выбраны близкими к ожидаемым на установке FACET-II: заряд пучка составлял $3 \mathrm{nC}$, среднеквадратичные диаметр и длина пучка составляли $400 \mathrm{~nm}$ и $1 \mu \mathrm{m}$ соответственно, энергия частиц $-10 \mathrm{GeV}$. Концентрация мишени изменялась в пределах от $10^{27} \mathrm{~m}^{-3}$ до $5 \times 10^{29} \mathrm{~m}^{-3}$, толщина — от 1 до $100 \mu \mathrm{m}$. Взаимодействие пучков с более протяжёнными мишенями не моделировались в настоящей работе, так как в таком случае существенными становятся также различные столкновительные (ядерные, кулоновские и т.п.) процессы, реализация которых отсутствует в коде QUILL.

Результаты моделирования показывают, что распространение пучка в мишени сопровождается образованием полости, практически полностью лишённой электронов и распространяющейся синхронно с пучком (рис. 1). В такой сильно нелинейной кильватерной волне образуются квазистатические радиальное электрическое 

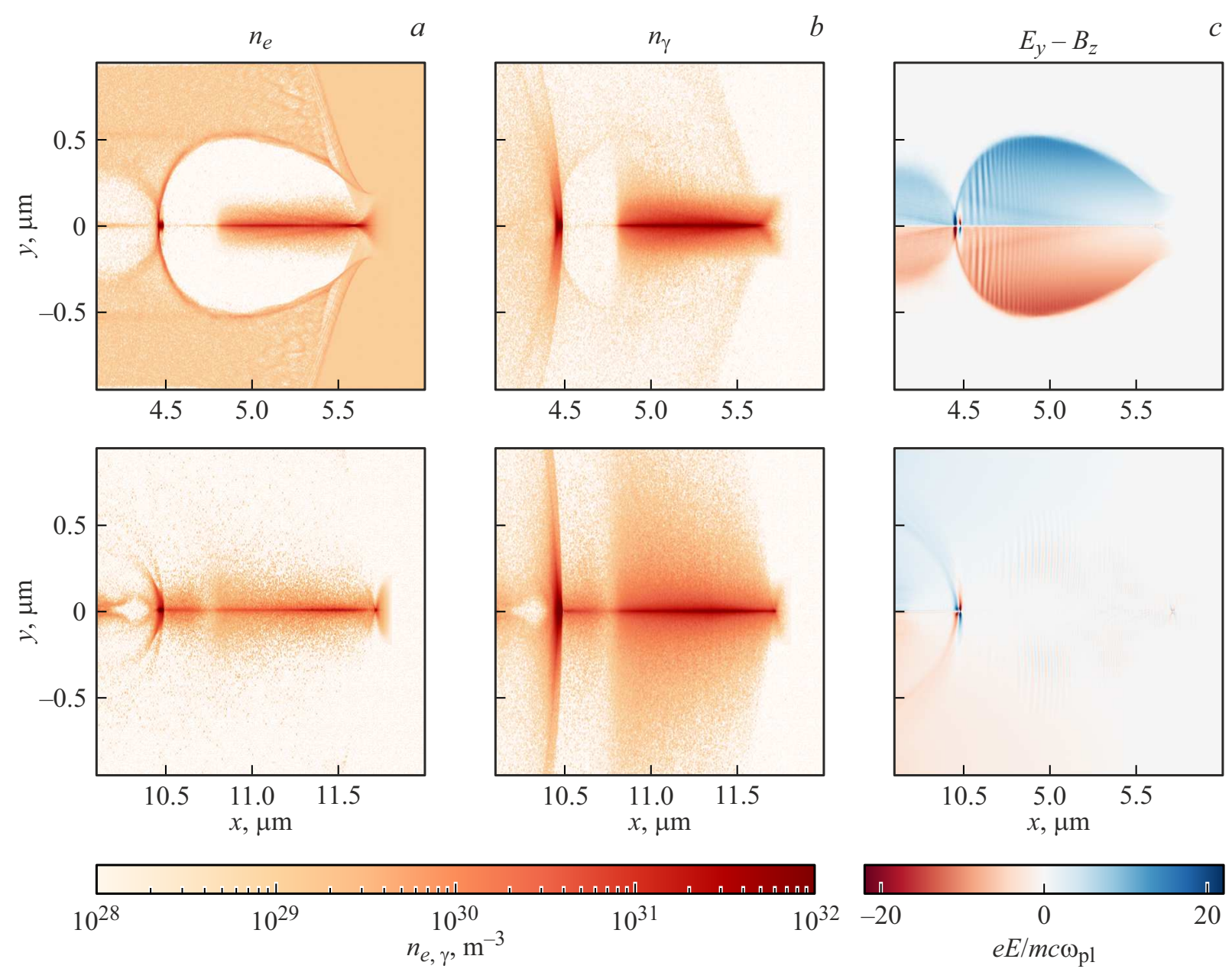

Рис. 1. (a) Распределение плотности электронов, $(b)$ плотности гамма-фотонов и $(c)$ поперечной силы $E_{y}-B_{z}$, действующей на электроны пучка, в моделировании распространения сильноточного пучка в твердотельной мишени с концентрацией $n_{e}=10^{29} \mathrm{~m}^{-3}$ и толщиной $10 \mu \mathrm{m}$. Верхний ряд соответствует проникновению пучка в мишень на глубину $4 \mu \mathrm{m}$, нижний ряд соответствует моменту выхода пучка из мишени.

и азимутальное магнитное поля, в которых частицы пучка совершают бетатронные колебания с частотой $\omega_{p l} / \sqrt{2 \gamma}$, где плазменная частота $\omega_{p l}$ соответствует невозмущённой концентрации электронов мишени, а $\gamma$ - мгновенное значение лоренц-фактора частицы. При таком движении излучение электронов некогерентно и имеет синхротронную природу. Генерируемый пучок гамма-квантов повторяет пространственное распределение электронов и обладает достаточно малой расходимостью. Гамма-излучение имеет широкий спектр с отсечкой на энергии начльных электронов $10 \mathrm{GeV}$, который практически не изменяется в процессе взаимодействия. Помимо потерь энергии на излучение, электроны пучка также замедляются за счёт продольного поля, генерируемого в плазменной полости. Однако для достаточно плотных мишеней данный эффект является значительно менее существенным по сравнению с потерями на излучение. Стоит отметить, что в результате формирования „баббла“ в его зад- ней части образуется вторичный пучок электронов, аналогично тому как это происходит в разреженной плазме. Электроны этого вторичного пучка находятся в ускоряющем продольном поле и также совершают бетатронные колебания и излучают. Результаты моделирования показывают, что энергия отсечки вторичного пучка не превышает $5 \mathrm{GeV}$, а доля суммарной энергии относительно энергии всего гамма-излучения не превышает $15 \%$.

Отметим, что недавно была предложена схожая схема для генерации ярких гамма-пучков, основанная на столкновении сильноточного пучка ультрарелятивистских частиц с последовательностью тонких металлических плёнок [25]. В данной конфигурации эффективное поле, действующее на электроны пучка, связанно с „отражением“ собственного поля пучка от тонкого плазменного слоя, и является по смыслу полем переходного излучения. Несмотря на различия в физическом механизме генерации гамма-квантов, эффективность ге- 


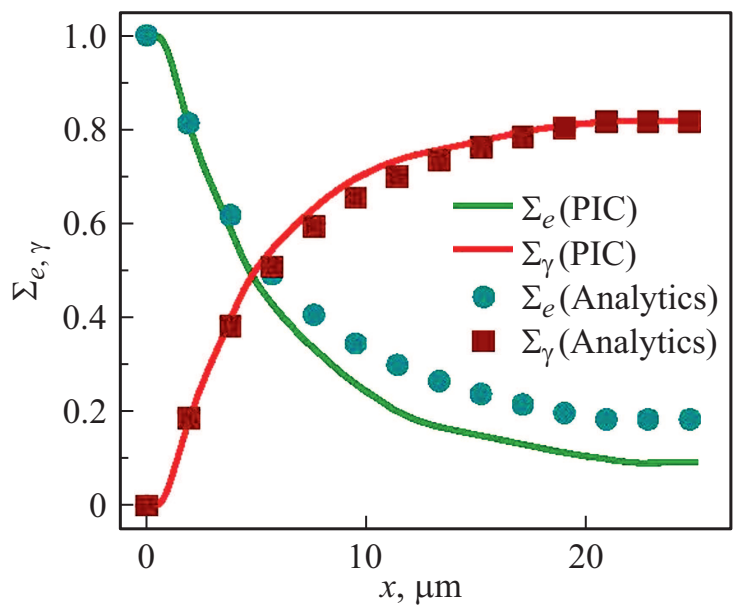

Рис. 2. Зависимость полной энергии электронов $\Sigma_{e}$ и фотонов $\Sigma_{\gamma}$ от времени, нормированная на полную начальную энергию электронов. Сплошные линии соответствуют результатам QED-PIC-моделирования, кружки и квадраты соответствуют оценке, рассчитанной с помощью выражения (4) и численного решения уравнений (1), (2).

нерации и спектр выходного гамма-излучения весьма похожи в конфигурации [25] и рассматриваемой нами конфигурации. Как было описано выше, электроны пучка совершают бетатронные колебания в поле сильно нелинейной кильватерной волны, структура которого описана, например, в публикации [26]. Усреднённые по периоду бетатронных осцилляций уравнения движения электрона в такой волне с учётом реакции излучения в квазиклассическом приближении [27-31] выглядят следующим образом (детальный вывод данных уравнений представлен, например, в публикациях [32,33]):

$$
\begin{gathered}
\frac{d \rho}{d t}=-\frac{\rho}{\gamma} \frac{1}{4 \pi} \int_{0}^{2 \pi} P\left(\frac{\rho \Gamma}{2 a_{S}}|\cos \phi|\right) \sin ^{2} \phi d \phi, \\
\frac{d \Gamma}{d t}=-\frac{1}{2 \pi} \int_{0}^{2 \pi} P\left(\frac{\rho \Gamma}{2 a_{S}}|\cos \phi|\right) d \phi,
\end{gathered}
$$

где $\rho$ - амплитуда бетатронных колебаний, $Г-$ энергия электрона, $a_{S}=m c^{2} / \hbar \omega_{p l}$. В данных уравнениях используется нормировка на плазменную частоту $\omega_{p l}$, соответствующую невозмущённой концентрации электронов мишени $n_{e}$ : время нормировано на $1 / \omega_{p l}$, координаты на $c / \omega_{p l}$, импульс - на $m c$, напряжённость электромагнитных полей - на $m c \omega_{p l} / e$, мощность - на $m c^{2} \omega_{p l}$. В классическом $\left(\chi_{0} \ll 1\right)$ и существенно квантовом $\left(\chi_{0} \gg 1\right)$ случаях, когда мощность радиационных потерь $P(\chi)$ является степенной функцией $\chi$, данные уравнения решаются аналитически:

$$
\frac{\Gamma(t)}{\gamma_{0}} \approx\left\{\begin{array}{l}
\left(1+0.625 P\left(\chi_{0}\right) t / \gamma_{0}\right)^{-4 / 5} \\
\left(1-0.149 P\left(\chi_{0}\right) t / \gamma_{0}\right)^{24 / 5}
\end{array}\right.
$$

где $\chi_{0}=r_{0} \gamma_{0} / 2 a_{S}, r_{0}-$ начальное отклонение электрона от оси пучка. Учитывая количество частиц, расположенных внутри мишени, можно окончательно определить зависимость полной энергии пучка от времени:

$$
\begin{aligned}
\Sigma_{e}(t) & =\Sigma_{0}-\int_{-2 \sigma_{x}}^{0} \int_{0}^{r_{b}}\left(\gamma_{0}-\gamma(x+c t)\right) \eta(r, x) \\
& \times \Theta(x+c t) 2 \pi r d r d x,
\end{aligned}
$$

где $\Sigma_{0}=N \gamma_{0}, N$ - число электронов в пучке, функция $\eta(x, r)=n_{b}(x, t) / N$ задаёт распределение заряда в пучке, $\Theta(x)$ - степ-функция Хевисайда. Пример сравнения такой оценки с результатами QED-PIC-моделирования представлен на рис. 2,3. Отметим, что в данной модели не учитывается наличие продольного поля в плазменной полости, которое дополнительно тормозит электроны пучка. Этим объясняется различие между оценкой энергии пучка (4) и её величиной в численном моделировании.

Отметим, что при использованных параметрах пучка даже при столкновении с плотной мишенью эффективность генерации электрон-позитронных пар из гаммаквантов достаточно мала, для того чтобы существенно повлиять на процесс столкновения. В связи с этим в настоящей работе образование электрон-позитронных пар не рассматривается детально.

Таким образом, с помощью полноразмерного трёхмерного численного моделирования нами была обнаружено, что при столкновении сильноточного пучка ультрарелятивистских электронов с протяжённой твердотельной мишенью генерируются два коротких сгустка гамма-фотонов. Первый из них связан с излучением электронами начального пучка, а второй - c излучением электронами, инжектированными в плазменную полость, создаваемую начальным пучком. При

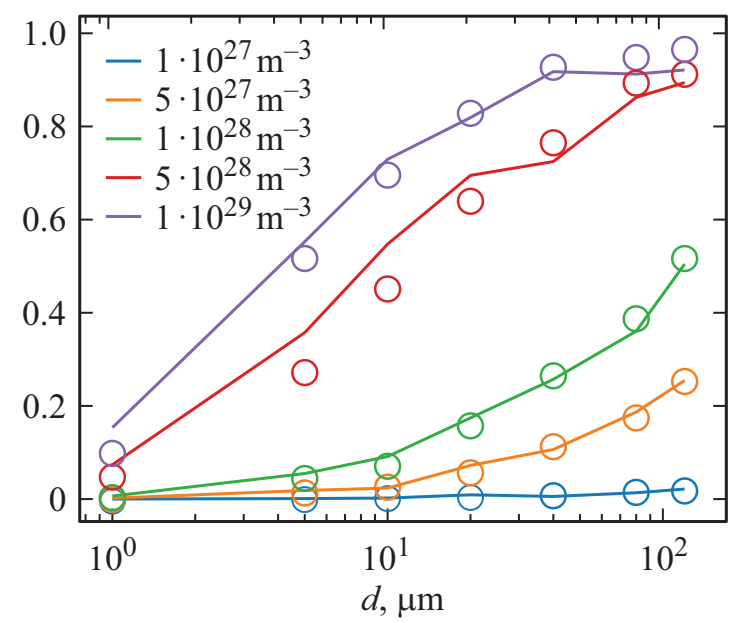

Рис. 3. Коэффициент конверсии энергии пучка электронов в энергию гамма-излучения в зависимости от концентрации и толщины мишени: сплошные линии - результаты QED-PICмоделирования, маркеры - аналитическая оценка (4). 
этом эффективность конверсии энергии пучка электронов в энергию гамма-фотонов может достигать 90\%. Изученная схема получения гамма-излучения является перспективной с точки зрения простоты экспериментальной реализации и крайне высокой эффективности.

\section{Финансирование работы}

Работа выполнена при поддержке Российского научного фонда (грант 20-12-00077).

\section{Конфликт интересов}

Авторы заявляют, что у них нет конфликта интересов.

\section{Список литературы}

[1] The Extreme Light Infrastructure [Электронный pecypc]. URL: https://www.eli-laser.eu

[2] Z. Gan, L. Yu, C. Wang, Y. Liu, Y. Xu, W. Li, S. Li, L. Yu, X. Wang, X. Liu, J. Chen, Y. Peng, L. Xu, B. Yao, X. Zhang, L. Chen, Y. Tang, X. Wang, D. Yin, X. Liang, Y. Leng, R. Li, Z. Xu. In: Progress in Ultrafast Intense Laser Science XVI, 199-217 (Springer) (2021).

DOI: $10.1007 / 978-3-030-75089-3 \_10$

[3] J. P. Zou, C. Le Blanc, D. N. Papadopoulos, G. Chériaux, P. Georges, G. Mennerat, F. Druon, L. Lecherbourg, A. Pellegrina, P. Ramirez, F. Giambruno, A. Frneaux, F. Leconte, D. Badarau, J.M. Boudenne, D. Fournet, T. Valloton, J.L. Paillard, J.L. Veray, M. Pina, P. Monot, J.P. Chambaret, P. Martin, F. Mathieu, P. Audebert, F. Amiranoff. High Power Laser Sci. Eng., 3 (e2) (2015). DOI: $10.1017 / \mathrm{hpl} .2014 .41$

[4] XCELS [Электронный ресурс].

URL: http://www.xcels.iapras.ru

[5] B. Shen, Z. Bu, J. Xu, T. Xu, L. Ji, R. Li, Z. Xu. Plasma Phys. Control. Fusion, 60 (4), 044002 (2018). DOI: $10.1088 / 1361-6587 / \mathrm{aaa} 7 \mathrm{fb}$

[6] C. Danson, C. Haefner, J. Bromage, T. Butcher, J. F. Chanteloup, E. A. Chowdhury, A. Galvanauskas, L. A. Gizzi, J. Hein, D. I. Hillier, N. W. Hopps, Y. Kato, E. A. Khazanov, R. Kodama, G. Korn, R. Li, Y. Li, J. Limpert, J. Ma, C. Hee Nam, D. Neely, D. Papadopoulos, R. R. Penman, L. Qian, J. J. Rocca, A. A. Shaykin, C. W. Siders, C. Spindloe, S. Szatm.ri, R. M. G. M. Trines, J. Zhu, P. Zhu,J. D. Zuegel. High Power Laser Sci. Eng., 7 (e54) (2019). DOI: 10.1017/hpl.2019.36

[7] C. Schroeder, E. Esarey, C. Geddes, C. Benedetti, W. Leemans. Phys. Rev. Accel. Beams, 13 (10), 101301 (2010). DOI: $10.1103 /$ physrevstab.13.101301

[8] Technical Design Report for the FACET-II Project at SLAC National Accelerator Laboratory (2016) [Электронный реcypc]. URL: http://www.osti.gov/servlets/purl/1340171/

[9] V. Yakimenko, S. Meuren, F. Del Gaudio, C. Baumann, A. Fedotov, F. Fiuza, T. Grismayer, M. Hogan, A. Pukhov, L. Silva, G. White. Phys. Rev. Lett., 122 (19), 190404 (2019). DOI: 10.1103/PhysRevLett.122.190404
[10] F. Del Gaudio, T. Grismayer, R. Fonseca, W. Mori, L. Silva. Phys. Rev. Accel. Beams, 22 (2), 023402 (2019). DOI: 10.1103/PhysRevAccelBeams.22.023402

[11] R.J. Noble. Nucl. Instrum. Methods Phys. Res. A, 256 (3), 427-433 (1987). DOI: 10.1016/0168-9002(87)90284-1

[12] R. Blankenbecler, S.D. Drell. Phys. Rev. D, 36 (1), 277 (1987). DOI: 10.1103/PhysRevD.36.277

[13] M. Bell, J.S. Bell. In: Quantum Mechanics, High Energy Physics Accelerators: Selected Papers Of John S Bell (With Commentary), ed. by M. Bell, K. Gottfried, M. Veltman (World Scientific, 1995), pp. 99-104. DOI: 10.1142/2611

[14] P. Chen, V.I. Telnov. Phys. Rev. Lett., 63 (17), 1796 (1989). DOI: 10.1103/PhysRevLett.63.1796

[15] J. Esberg, U. Uggerhøj, B. Dalena, D. Schulte. Phys. Rev. Accel. Beams, 17 (5), 051003, (2014). DOI: 10.1103/PhysRevSTAB.17.051003

[16] M. Tamburini, S. Meuren. Phys. Rev. D, 104 (9), L091903 (2020). DOI: 10.1103/PhysRevD.104.L091903

[17] M. Filipovic, C. Baumann, A.M. Pukhov, A.S. Samsonov, I.Yu. Kostyukov. Quantum Electron., 51 (9), 807-811 (2021). DOI: 10.1070/qel17606

[18] J. Rosenzweig, B. Breizman, T. Katsouleas, J. Su. Phys. Rev. A, 44 (10), R6189 (1991). DOI: 10.1103/PhysRevA.44.R6189

[19] A. Pukhov J. Meyer-ter Vehn. Appl. Phys. B, 74 (4), 355-361 (2002). DOI: $10.1007 / \mathrm{s} 003400200795$

[20] QUILL code [Электронный ресурс]. URL: https://github.com/QUILL-PIC/Quill

[21] A. Samsonov, A. Pukhov, I. Kostyukov. J. Phys.: Conf. Ser., 1692 (1), 012002 (2020). DOI: $10.1088 / 1742-6596 / 1692 / 1 / 012002$

[22] C.K. Birdsall, A.B. Langdon. Plasma physics via computer simulation (CRC press, 2004).

[23] M.D. Meyers, C.K. Huang, Y. Zeng, S.A. Yi, B.J. Albright. J. Comput. Phys., 297, 565-583 (2015). DOI 10.1016/j.jcp.2015.05.037

[24] A. Blinne, D. Schinkel, S. Kuschel, N. Elkina, S. Rykovanov, M. Zepf. Comput. Phys. Comm., 224, 273-281 (2018). DOI: 10.1016/j.cpc.2017.10.010

[25] A. Sampath, X. Davoine, S. Corde, L. Gremillet, M. Gilljohann, M. Sangal, C. Keitel, R. Ariniello, J. Cary, H. Ekerfelt, C. Emma, F. Fiuza, H. Fujii, M. Hogan, C. Joshi, A. Knetsch, O. Kononenko, V. Lee, M. Litos, K. Marsh, Z. Nie, B. O.Shea, J. Peterson, P. Claveria, D. Storey, Y. Wu, X. Xu, C. Zhang, M. Tamburini. Phys. Rev. Lett., 126 (6), 064801 (2021). DOI: 10.1103/PhysRevLett.126.064801

[26] I. Kostyukov, A. Pukhov, S. Kiselev. Phys. Plasmas, 11 (11), 5256-5264 (2004). DOI: 10.1063/1.1799371

[27] J.G. Kirk, A. Bell, I. Arka. Plasma Phys. Control. Fusion, 51 (8), 085008, (2009). DOI: $10.1088 / 0741-3335 / 51 / 8 / 085008$

[28] S. Bulanov, C. Schroeder, E. Esarey, W. Leemans. Phys. Rev. A, 87 (6), 062110 (2013). DOI: $10.1103 /$ PhysRevA.87.062110

[29] T.Z. Esirkepov, S.S. Bulanov, J.K. Koga, M. Kando, K. Kondo, N.N. Rosanov, G. Korn, S.V. Bulanov. Phys. Lett. A, 379 (36), 2044 (2015). DOI: 10.1016/j.physleta.2015.06.017

[30] F. Niel, C. Riconda, F. Amiranoff, R. Duclous, M. Grech. Phys. Rev. E, 97 (4), 043209 (2018). DOI: $10.1103 /$ physreve. 97.043209 
[31] A. Gonoskov, T. Blackburn, M. Marklund, S. Bulanov. Charged particle motion and radiation in strong electromagnetic fields [Электронный pecypc]. URL: https://arxiv.org/abs/2107.02161

[32] A.S. Samsonov, E.N. Nerush, I.Y. Kostyukov, M. Filipovic, C. Baumann, A. Pukhov. New J. Phys., 23 (10), 103040 (2021). DOI: 10.1088/1367-2630/ac2e84

[33] A.A. Golovanov, E.N. Nerush, I.Yu. Kostyukov. Radiation reaction-dominated regime of wakefield acceleration [Электронный ресурc]. URL: https://arxiv.org/abs/2109.04866 